\section{Blinding in pharmacological trials: the devil is in the details}

\author{
Mandy Wan, ${ }^{1}$ Mine Orlu-Gul, ${ }^{2}$ Helene Legay, ${ }^{3}$ Catherine Tuleu ${ }^{2}$
}

While the methodological principle of 'blinding' for minimising bias in randomised controlled trials (RCT) is widely debated and accepted, rarely is this depth of thinking applied to its correct handling with respect to design, reporting and analysis. Beyond the general comprehension of the definition of the word, the practical aspects of establishing blinding in investigator-initiated pharmacological trials are often grossly underestimated, ${ }^{1}$ and very much an afterthought. The resulting inadequacy can lead to trial delayed and increased costs, ${ }^{1}$ and can also potentially place the entire trial at risk.

It is worth nothing that reporting of blinding methodology is often absent or of low quality in published articles of RCTs. $^{2}{ }^{3}$ Thus, in such context, when these fundamental details of trial design are inadequately reported, ${ }^{2} 3$ it becomes easier to see why it should be of no surprise that there are, equally, very few published commentaries addressing the challenges of blinding that those embarking on pharmacological trials face. So, it would seem that little is available, at least in biomedical literature, to guide investigators on this particular methodological aspect. A review of RCTs published over 15 years in this journal identified only 176 RCTs in children involving pharmaceutical interventions ${ }^{4}$; the authors suggested that difficulties in obtaining adequate placebos without the collaboration of pharmaceutical companies may have contributed to the low number. ${ }^{4}$ Our experience over the years has also highlighted, among investigators, a deficiency in the awareness of how pharmaceutical ramification can constrain trial design and its validity. ${ }^{5}$ There is no denying that the

\footnotetext{
${ }^{1}$ National Institute for Health Research-Medicines for Children Research Network-London \& South East, Evelina London Children's Hospital, Guy's and St Thomas NHS Foundation Trust, King's Health Partners, London, UK: ${ }^{2}$ Department of Pharmaceutics, UCL School of Pharmacy, Centre for Paediatric Pharmacy Research, London, UK; ${ }^{3}$ Faculté Des Sciences Pharmaceutiques et Biologiques de Lyon, Université Claude Bernard, Lyon, France

Correspondence to Mandy Wan, National Institute for Health Research, Medicines for Children Research Network - London \& South East, Guy's and St Thomas' NHS Foundation Trust, St Thomas' Hospital, St Thomas' House, 5th Floor, London SE1 7EH, UK; mandy.wan@gstt.nhs.uk
}

science underpinning blinding is very much pharmaceutical based, and may be technical at times, but part of this paucity of knowledge is undoubtedly due to a certain lack of recognition for the pharmaceutical properties of a medicine. This article, written with clinicians in mind, presents a discussion on the practical considerations for blinding in paediatric pharmacological trials, with the aim to facilitate paediatricians in improving the success and timely delivery of blinded RCTs in children. Furthermore, this article aims to encourage detailed disclosure of blinding methodology in clinical trial reporting.

\section{BLINDING}

Blinding in clinical trials refers to the process of withholding information about the assigned treatment from specific groups of individuals. The first blinded experiment was conducted by Benjamin Franklin who literally blindfolded participants to shield them from knowledge in their assessments of the therapeutic claims made for applying mesmerism. Quite understandably, the use of blindfolds is less favourable today. Instead, identical-appearing treatments, be it matching placebo or masked active comparator, are important tools in modern-day pharmacological research.

When performed correctly, blinding is intended to minimise the occurrence of conscious and unconscious bias in the conduct and interpretation of a trial until all such opportunities for bias have passed. The biases associated with prior knowledge of treatment assignment are well known, and the benefits of blinding have been presented elsewhere ${ }^{3} 7$ Indeed, it is acknowledged that the relevance of blinding will vary according to the clinical trial context. In general, blinding of participants, healthcare providers and outcome assessors is considered important in explanatory trials, where the primary focus is to determine the efficacy of an intervention under ideal circumstances. ${ }^{8}$ By contrast, in pragmatic trials, as in the real-world delivery of care, blinding of participants and healthcare providers are sometimes considered not necessary, so as to render the findings more applicable to usual care setting. ${ }^{8-10}$ Blinding is also particularly important when outcome measures involve some subjectivity, and becomes less so to reduce observer bias for objective criteria. ${ }^{7}$ However, even then, the lack of participant or healthcare provider blinding can lead to other problems, such as differential attrition and cointervention bias, which can likewise influence the assessment of clinical trial outcomes. $^{7} 10$

\section{BLINDING WITH PLACEBOS}

The use of placebo in RCTs would appear as a seemingly simple experimental scheme. Such perception is so deeply embedded that we often implicitly accept clinical trial reporting with rather loose descriptions of blinding procedures as adequate indication for the success of blinding. $^{2}{ }^{3}$ Rarely do we ask ourselves any questions concerning the placebo, nor do we think much, if at all, about the work involved in producing them. The fact is that the seemingly simple concept does not necessarily hold true for obtaining placebo supply. One cannot just purchase matching placebo in an 'off-the-shelf' manner; its provision needs to be specific to each trial and the challenge is in the word 'matching'. To achieve its purpose, one must ensure the placebo matches the sensory specifications to that of the medicine under test. The ability to maintain blindness throughout the complete clinical trial must focus on the visual aspects of the product, and should also take into consideration the other human senses. The relative importance of these specifications will very much depend on the route of administration and the dosage form concerned; this may include: shape, size, colour, texture, weight, taste, smell ${ }^{11}$ and not merely a 'sugar pill'.

An appropriate source for supply would be the original manufacturer of the medicine. After all, they would have manufactured placebo for their own marketing authorisation trials, and have all the required technical data on the manufacturing and analytical methods to support clinical trial application. However, for many pharmaceutical companies, the little incentive to manufacture placebos on the comparatively limited quantity required by most clinical trials is often disappointing to the independent researchers. ${ }^{1} 1213$ Even if one is fortunate enough to have agreed a supply, commercial influence over protocol design, trial results and publication can be somewhat disheartening. ${ }^{12} 14$

Short of compromising the study design with an unblinded trial, an alternative approach is to have the matching placebo manufactured elsewhere. While it would 
be logical to use the same ingredients (excipients) in the placebo formulation as those in its active counterpart (without the active drug), such approach does not always guarantee a satisfactory level of blinding. First, blinding is particularly challenging where the actual drug itself has a characteristic taste or colour, necessitating the testing and addition of new excipients to the placebo formulation in order to achieve sensory equivalent. ${ }^{15} 16$ Second, and rather more problematically, is when such addition is unsatisfactory; in these circumstances, it may be necessary to reformulate the active treatment from its licensed form (eg, the addition of a new excipient to mask the taste of the active drug), to what is in effect manufacturing of a new product. The decision to adopt such blinding strategy should, of course, never be taken lightly.

To allow for variable dose delivery as a result of age/weight/surface area-based dosing, and to facilitate ease of administration, liquid oral dosage form is often required in the treatment of children, making blinding liquid formulation a more frequent issue in paediatric setting. Compared with tablets and capsules, the sensory characteristics of taste and smell are more pronounced, yet, challenging to match. Even simple flavourings, such as strawberry flavour, can vary in taste depending on the manufacturer, and thus requiring the need to perform taste assessment studies. There is also the additional physical attribute of viscosity which one must consider. Hence, some degree of formulation development work is almost always needed in developing a placebo liquid formulation.

Likewise, for placebos intended for topical use, the property of $\mathrm{pH}$ deserves particular attention; the differences in $\mathrm{pH}$ between the two products may lead to local irritation in one group only, and therefore distinct side-effects profile will effectively unblind the treatment arm and biased assessments of endpoints may result.

Even if developing the placebo formulation was straightforward, difficulties can arise with uniquely shaped tablets or products with specific markings, ${ }^{1}$ where the complexity of matching these shapes and proprietary designs may require specialised equipment. The challenge is not simply a matter of cost relating to the equipment purchase, but also the fact that the installation of new equipment will necessitate a lengthy programme of qualification and validation work. Less obviously, perhaps, is the possible issue of infringement on trademark law. As far as copying goes, certain imprints or markings on tablets are registered trademarks, and therefore exclusive rights are bestowed upon the trademark owner, the drug's original manufacturer. Consequently, third-party placebo manufacturing is not always possible.

\section{BLINDING OF ACTIVE COMPARATORS}

To many, over-encapsulation may appear to be the ubiquitous solution to blinding solid oral formulations, and indeed, such a technique can be very effective. As the term implies, over-encapsulation is basically hiding a tablet or capsule inside an opaque capsule shell (often involving the addition of a backfilled excipient to prevent rattling), so that the contents are concealed, producing products that are visually identical. This method of blinding eliminates the need for matching placebo manufacturing, and is particularly useful for active-controlled trials requiring comparator blinding. However, overencapsulation increases the size of the original dosage form, potentially making administration more difficult. The use of such blinding method in a trial involving the younger subset of the paediatric population may therefore be problematic. More importantly, one must remember that a medicine is made up of ingredients other than the active drug, and the effects of the drug are, in part, dependent on the properties and proportions of these other components. Thus, it is the dosage form in which the active drug is administered that determines the overall therapeutic efficacy and safety of the drug. As overencapsulation brings new ingredients into the question, and effectively creating a new dosage form, demonstrating equivalence between the encapsulated and original products becomes a prerequisite for ensuring study result integrity. ${ }^{9} 17$

Alternatively, blinding of two substantially different looking treatments may be achieved by bespoke manufacturing both treatments from their respective active pharmaceutical ingredients into either capsules or tablets. ${ }^{9}$ With this method, the visual appearance of the two treatments can be controlled from the outset, but again, there remains the question of equivalent bioavailability, as well as the operational challenges in manufacturing two formulations within the resource constraints imposed by finite research funding.

Suppose we now have a blinded activecontrolled comparator trial of two commercially available liquid formulations. This clearly differs from the case with tablets or capsules, whereby over- encapsulation can potentially provide the solution to the problem. A sensible approach would be to consider the doubledummy blinding strategy. With this approach, the comparator doesn't need to match the test product. Instead, a placebo matching the test product and a second placebo matching the comparator product are used. Although such a study design may be more pragmatic, it does have some drawbacks. On the one hand, there are considerable technical difficulties, as discussed above, with manufacturing placebo for liquid formulation. As two matching placebos are now needed, this would require additional research expense and time. On the other hand, the doubledummy design requires participants to take two study medications; in cases where the medicines concerned require multiple daily doses, this raises the question of the potential risk of non-compliance.

For infusion therapy, blinding can translate into a somewhat cumbersome setup. Some researchers have used opaque tubing and the covering of infusion bag with plastic sleeve to mask its content. ${ }^{18}$ We question the robustness of such blinding method, where we believe that the risk of unblinding through routine handling of the infusion administration set is substantial. Moreover, when visual inspection of the infusion bag and its administration set is a standard safety precaution measure for nursing staff when administering infusion therapy, the feasibility and acceptability of such blinding technique may be limited in practice to allow the trial to be delivered successfully. In these situations, researchers must acknowledge this reality, accepting the unblinded status of those administering the treatment. The use of unblinded personnel, though imperfect, does not necessarily lead to an unblinded trial. By limiting the activity of the unblinded personnel to that of treatment administration only, it remains possible to blind other key trial personnel, and not limited to only outcome assessors. Nonetheless, researchers should anticipate that maintaining blinding by such methodology will be labour intensive, and that guarding against inappropriate unblinding must be a high priority.

\section{THE 'INERT' INGREDIENTS}

There is certainly a need to consider the suitability of an excipient for use in placebo manufacturing or in comparator products masking with respect to its safety. Indeed, the basic considerations will not be different to that of marketed medicines, with even greater scrutiny in a paediatric setting in view of the potential 
risk of more pronounced safety implications. Risk assessments of excipients used in the context of blinding still apply, and on the whole, those with identified risks relevant to the target age groups concerned should be avoided, as their use cannot be reasonably justified for the sole purpose of blinding.

Moreover, the use of any excipient requires additional concern considering the fact that excipients have proved to be anything but inert, ${ }^{19}$ and these possible specific effects represent a potential confounding factor that may be vital to interpreting the study results. The appropriateness of using nebulised saline as a placebo in bronchodilator studies assessing symptoms relief involving patients with respiratory problem has been questioned. $^{20}$ Similarly, placebos of corn oil or olive oil in cardiac trials have raised doubts. $^{21}$ The question of under what circumstances it is suitable, if ever, to use a particular excipient will depend on our current knowledge, and this may change with advances in scientific research. With this in mind, the disclosure of placebo composition in clinical trial reporting, at least in cases where there are differences in excipients between the two experimental arms, becomes ever so important should further excipient data emerge at a later date.

\section{PACKAGING}

There is little merit in creating a matching placebo or masking a comparator, when for example, the active is contained in a silver aluminium blister strip whereas white plastic blister has been used to package the placebo. The requirement for matching must go beyond the actual products and extends to all associated packaging and labelling. On occasions, the need for repackaging of medicines cannot be avoided and its implications must be reviewed at the outset.

The need for repackaging may not always be apparent at the time when the pharmaceutical company agreed to provide the clinical trial supply. Amidst the excitement of agreeing the supply, one must request for detailed information on the packaging to assess its suitability for the trial. Medicines provided in its commercial form are often packaged in a branded manner and as such cannot be copied. Other than the additional time and cost for depackaging and repackaging activities, the impact of these undertakings on product integrity and stability needs to be reviewed. The original manufacturer's stability data supports only the medicine in its original packaging, and unless equivalent or more protective packaging is used, which itself may be difficult to determine, a reduction in the medicine expiry date cannot be avoided. Although a reduction in the expiry date from 36 to 24 months may have little bearing for a trial of 12 months duration, the effect of such reduction on a 30 months trial can be very costly.

\section{REPORTING}

In light of the challenges discussed above, it is surprising that such topics have had so little mention in most commentaries. Then again, despite being included in the minimum set of recommendations in the Consolidated Standards of Reporting Trials (CONSORT) statement, ${ }^{22}$ the reporting of the blinding status of key trial persons, and of the blinding procedures, is still frequently incomplete or missing. ${ }^{23}$ Most of all, when the reporting of basic information such as details on the dosage form of medicine is frequently absent in published articles, ${ }^{24}$ it does beg the question as to whether readers have simply assumed that blinding has been appropriately controlled for biases without actually knowing how well it worked or even if it worked at all.

While it would be inappropriate to assume inadequate blinding merely on the basis of inadequate reporting, equally, the assumption of adequate blinding when reporting is absent or inadequate cannot be justified. If most researchers supposedly comprehend the importance of establishing blinding, then it is only expected that they would also want to know whether blinding was executed successfully. We recognised that there are methodological uncertainties surrounding testing the success of blinding, ${ }^{1} 24$ but nonetheless, we support greater disclosure and transparency in blinding methodology reporting in a manner that reflects pharmaceutical considerations. There certainly should be explicit reporting on who was blinded, with additional information on the methods involved in introducing and maintaining blinding. ${ }^{3} 7$ Where feasible, we also strongly encourage the conduct and reporting of pretrial evaluation of the identity of the two compared treatments. ${ }^{3} 25$

We recognised that blinding of all key trial personnel is not always technically or practically possible, and on occasion, the cost of blinding is such that the trial is no longer feasible even with generous increase in funding. Do we then simply disregard blinding? The issue is not whether blinding should be disregarded, but investigators need to carefully assess how much bias might ensue owing to absence of blinding, and to explore alternative methods of trial design to minimise the risk of bias in the trial. More importantly, they should provide clear justifications for their decisions, and report any speculation on the effect of any factors arising from the course of the trial which may impact on the outcome measures. Clinical trials, even when most personnel are unblinded, can remain methodologically sound in terms of controlling selection bias through proper randomisation and adequate allocation concealment. However, we, like others, take the view that it is almost always possible to ensure that the outcome assessor in a trial is blinded. ${ }^{3}$ We strongly encourage this aspect of blinding to be implemented, and if not done, the rationale should be clearly reported.

\section{DISCUSSION}

The concept of blinding may appear relatively simple, but in reality, substantial practical difficulties can arise in constructing apparently identical treatment. Just as you would always discuss with an experienced statistician when designing a trial, for example, the need for stratification in randomisation or the population SD in sample size determination, the same level of detailed discussion is needed with respect to blinding. Determining the most appropriate blinding strategy is often complex; as highlighted by the recurring theme of this article, significant timing and cost is involved. As such, blinding strategy deserves particular attention from investigators at the earliest stages of trial planning, preferably before seeking funding.

We recommend that investigators take a balance approach to blinding strategy with consideration of a risk-based assessment of clinical, pharmaceutical, logistical and resource factors. Engaging with experienced individuals, such as research pharmacists, and initiating dialogue on formulations and blinding early in the trial design phase can help to avoid unworkable blinding options, as well as to ensure the work required for blinding is timely delivered. ${ }^{1}$ Considering the challenges discussed, we further recommend funding bodies to consider making pharmaceutical expert review a condition of public funding for pharmacological RCTs. At the very least, editors of biomedical journals should mandate explicit blinding reporting be part of articles considered for publication. It is only through better reporting and critical analysis that 
we can build our body of knowledge on blinding methodology.

An article of this size cannot possibly address the different approaches to blind different dosage forms or the regulatory framework governing pharmaceutical manufacturing. Rather, the aim is to introduce researchers to some of the key pharmaceutical concepts and associated vocabularies. Blinding should, under no circumstances, be seen as a means to an end, when in fact, pharmaceutical properties of the medicine should drive the choice of blinding method which, in part, will inform the design and operational delivery of the trial.

Contributors MW-conception, literature search, drafting of the manuscript, final approval of manuscript; MOG - conception, literature search, critical revision of manuscript; $\mathrm{HL}$ - design, literature search, critical revision of manuscript; CT—conception, critical revision of manuscript.

\section{Competing interests None.}

Provenance and peer review Not commissioned; externally peer reviewed.

\section{(6) OPEN ACCESS}

Open Access This is an Open Access article distributed in accordance with the Creative Commons Attribution Non Commercial (CC BY-NC 3.0) license, which permits others to distribute, remix, adapt, build upon this work non-commercially, and license their derivative works on different terms, provided the original work is properly cited and the use is non-commercial. See: http://creativecommons.org/ licenses/by-nc/3.0/

To cite Wan M, Orlu-Gul M, Legay $H$, et al. Arch Dis Child Published Online First: [please include Day Month Year] doi:10.1136/archdischild-2013-304037

Received 9 March 2013

Revised 6 June 2013

Accepted 3 July 2013

Arch Dis Child 2013;98:656-659.

doi:10.1136/archdischild-2013-304037

\section{REFERENCES}

1 Whitham D, Silcocks $P$, Whitehouse W, et al. An example of problems that arise from clinical trials and how to avoid them. Pharm J 2009;283:129-30.

2 Haahr MT, Hróbjartsson A. Who is blinded in randomized clinical trials? A study of 200 trials and a survey of authors. Clin Trials 2006;3:360-5.

3 Hróbjartsson A, Boutron I. Blinding in randomized clinical trials: imposed impartiality. Clin Pharmacol Ther 2011;90:732-6.

4 Campbell T, Surrey SA, Royle EM. A review of randomized controlled trials published in Archives of Disease of Childhood from 1982-96. Arch Dis Child 1998;79:192-7.

5 Jefferson T, Jones MA, Doshi P, et al. Neuraminidase inhibitors for preventing and treating influenza in healthy adults and children. Cochrane Database Syst Rev 2012;(1):CD008965.

6 Karlowski TR, Chalmers TC, Frenkel LD, et al. Ascrobic acid for the common cold. A prophylactic and therapeutic trial. JAMA 1975;231:1038-42.

7 Schulz KF, Grimes DA. Blinding in randomised trials: hiding who got what. Lancet 2002;359:696-700.

8 Sackett DL. Explanatory and pragmatic clinical trials: a primer and application to a recent asthma trial. Pol Arch Med Wewn 2011;121:259-63.

9 Martin BK, Meinert CL, Breitner JC, et al. Double placebo design in a prevention trial for Alzheimer's disease. Control Clin Trials 2002;23:93-9.

10 Zwarenstein M, Treweek S, Gagnier JJ, et al. Improving the reporting of pragmatic trials: an extension of the CONSORT statement. BMJ 2008:337:a2390.

11 Joyce $C$. Placebos and other comparative treatments. Br J Clin Pharmac 1982:13:313-18.
12 Christensen M, Knop FK. The unobtainable placebo: control of independent clinical research by industry? Lancet 2012;379:30

13 Curfman GD, Morrissey S, Drazen JM. Products at risk. N Engl J Med 2010;363:1763.

14 Lenney W, Perry S, Price D. Clinical trials and tribulations: the MASCOT study. Thorax 2011;66:457-8

15 Mahalanabis D, Lahiri M, Paul D, et al. Randomized, double-blind, placebo-controlled clinical trial of the efficacy of treatment with zinc or vitamin A in infants and young children with severe acute lower respiratory infection. Am J Clin Nutr 2004;79:430-6.

16 Morganroth J, Dimarco JP, Anzueto A, et al. A randomized trial comparing the cardiac rhythm safety of moxifloxacin vs levofloxacin in elderly patients hospitalized with community-acquired pneumonia. Chest 2005:128:3398-406.

17 European Medicines Agency. Guideline on the requirements to the chemical and pharmaceutical quality documentations concerning investigation medicinal products in clinical trials. London: Committee for Medicinal Products for Human Use (CHMP), 2006.

18 Boutron I. Methods of blinding in reports of randomized controlled trials assessing pharmacologic treatments: a systematic review. PLoS Med 2006;3:e425.

19 What are excipients doing in medicinal products? DTB 2009:47:81-4.

20 Khan SY, O'Driscoll BR. Is nebulized saline a placebo in COPD? BMC Pulm Med 2004:30:9-84.

21 Golomb BA. Paradox of placebo effect. Nature 1995:375:530

22 Schulz KF, Altman DG, Moher D; for the CONSORT Group. CONSORT 2010 Statement: updated guidelines for reporting parallel group randomised trials. Ann Int Med 2010;152:726-32.

23 Pandit S, Shah U, Kirby DJ, et al. Inappropriate oral formulations and information in paediatric trials. Arch Dis Child 2010;95:754-6.

24 Hróbjartsson A, Forfang E, Haahr MT, et al. Blinded trials taken to the test: an analysis of randomized clinical trials that report tests for the success of blinding. Int J Epidemol 2007:36:654-63.

25 Walter SD, Awasthi S, Jeyaseelan L. Pre-trial evaluation of the potential for unblinding in drug trials: a prototype example. Contemp Clin Trials 2005;26:59-468. 